\title{
ON STARLIKE AND CLOSE-TO-CONVEX FUNCTIONS
}

\author{
By CHRISTIAN POMMERENKE
}

[Received 20 March 1962]

\section{The maximum modulus}

A Function $f(z)=a_{1} z+\ldots$ is called starlike in $|z|<1$ if it is analytic in $|z|<1$ and maps $|z|<1$ onto a region starlike with respect to 0 . Let

$$
M(r)=\max _{|z|=r}|f(z)|
$$

be the maximum modulus of $f(z)$. The author has shown ((8) Theorem 1) that

exists and that

$$
\alpha=\lim _{r \rightarrow 1} \log M(r) / \log \frac{1}{1-r}
$$

$$
M(r) \geqq \frac{\left|a_{1}\right|}{4} \frac{r}{(1-r)^{\alpha}} .
$$

We shall call $\alpha$ the order of $f(z)$. For every $\theta$,

$$
V(\theta)=\lim _{r \rightarrow 1} \arg f\left(r e^{i \theta}\right)
$$

exists, and $V(\theta)$ is monotone increasing. Hence $V(\theta)$ is continuous with the exception of an at most enumerable number of jump discontinuities. Hence there is a greatest jump. This greatest jump has the height $\pi \alpha$ ( (8) Theorem 1).

Theorem 1. Let $f(z)$ be starlike in $|z|<1$ and let $\alpha(0 \leqslant \alpha \leqslant 1)$ be the order of $f(z)$. Then

$$
\alpha=\lim _{r \rightarrow 1}(1-r) \frac{M^{\prime}(r)}{M(r)}
$$

where $M^{\prime}(r)$ is the left derivative.

For every analytic function, the maximum modulus $M(r)$ is piecewise analytic in $0 \leqslant r<1$. Hence the left derivative always exists. We can choose $\zeta$ on $|\zeta|=r$ such that $|f(\zeta)|=M(r)$ and (see for instance (4))

$$
\zeta \frac{f^{\prime}(\zeta)}{f(\zeta)}=r \frac{M^{\prime}(r)}{M(r)}
$$

Proof. 1. Suppose (4) were false. Then there would exist a sequence $\left\{r_{k}\right\}$ with $r_{k} \rightarrow 1$ such that

$$
\left(1-r_{k}\right) \frac{M^{\prime}\left(r_{k}\right)}{M\left(r_{k}\right)}+\alpha
$$

Proc. London Math. Soc. (3) 13 (1963) 290-304 
Let $M\left(r_{k}\right)=f\left(r_{k} e^{i \theta_{k}}\right)$, where $\zeta_{k}=r_{k} e^{i \theta_{k}}$ satisfies (5). We may assume that $\left\{\theta_{k}\right\}$ converges. Using a suitable normalization of $f(z)$ we may assume that $\theta_{l:} \rightarrow 0$.

If $V(\theta)$ is defined by (3) then (see for instance (7))

$$
f(z)=a_{1} z \exp \left(\frac{1}{\pi} \int_{0}^{2 \pi} \log \frac{1}{1-e^{-i t} z} d V(t)\right) .
$$

From this representation it follows that (see the formula before (7) in (7))

$$
\log \left|f\left(r e^{i \theta}\right)\right| \leqq \log \left|a_{1}\right|+\frac{2 \pi}{\sin ^{2} \eta}+\frac{1}{\pi}(V(\theta+\eta)-V(\theta-\eta)) \log \frac{1+r}{1-r}
$$

for $0<\eta<\pi / 2$. Hence (the numbers $K_{j}$ will depend only on $f$ and on the variables displayed)

$$
\log M\left(r_{k}\right) \leqq K_{1}(\eta)+\frac{1}{\pi}\left(V\left(\theta_{k}+\eta\right)-V\left(\theta_{k}-\eta\right)\right) \log \frac{1}{1-r_{k}}
$$

If $V(\theta)$ has the jump $\pi \alpha^{*}$ at $\theta=0$ then, for every $\epsilon>0$, there is an $h_{0}>0$ such that $V(h)-V(-h)<\pi\left(\alpha^{*}+\epsilon\right)$ for $0 \leqslant h<h_{0}$. Since $V(\theta)$ is monotone it follows that $V\left(\theta_{k}+\eta\right)-V\left(\theta_{k}-\eta\right)<\pi\left(\alpha^{*}+\epsilon\right)$ if $\eta>0$ is sufficiently small and $k$ large. Taken together with (2) this implies that

$$
K_{2}+\alpha \log \frac{1}{1-r_{k}} \leqq \log M\left(r_{k}\right) \leqq K_{3}(\epsilon)+\left(\alpha^{*}+\epsilon\right) \log \frac{1}{1-r_{k}}
$$

for large $k$. Making $k \rightarrow \infty$ we see that $\alpha \leqq \alpha^{*}+\epsilon$; hence $\alpha \leqq \alpha^{*}$. On the other hand, $\pi \alpha$ is the greatest jump of $V(\theta)$. Therefore $\alpha^{*}=\alpha$, and $V(\theta)$ has a jump of height $\pi \alpha$ at 0 .

2. We assume now that $\alpha>0$. We want to show that $\theta_{l k} /\left(1-r_{k}\right)$ remains bounded as $k \rightarrow \infty$. If this is not true we can assume that for instance $\theta_{k}>0$ and that $\theta_{k} /\left(1-r_{k}\right) \rightarrow+\infty$. From (7) we obtain

$$
\log \left|\frac{f\left(r_{k}\right)}{f\left(r_{k} e^{\left.i \theta_{k}\right)}\right.}\right|=\frac{1}{2 \pi} \int_{-\pi}^{\pi} \log \frac{1-2 r_{k} \cos \left(t-\theta_{k}\right)+r_{k}^{2}}{1-2 r_{k} \cos t+r_{k}{ }^{2}} d V(t) .
$$

We can write $V(t)=\alpha S(t)+V^{*}(t)$, where $S(t)$ is constant in $-\pi \leqq t \leqq \pi$ except for a jump of height $\pi$ at $t=0$, and $V^{*}(t)$ is monotone and continuous at $t=0$. Then it follows that

$$
\begin{aligned}
\log \left|\frac{f\left(r_{k}\right)}{f\left(r_{k} e^{i \theta_{k}}\right)}\right|= & \frac{\alpha}{2} \log \frac{1-2 r_{k} \cos \theta_{k}+r_{k}^{2}}{\left(1-r_{k}\right)^{2}} \\
& -\frac{1}{2 \pi} \int_{-\pi}^{\pi} \log \frac{1-2 r_{k} \cos t+r_{k}^{2}}{1-2 r_{k} \cos \left(t-\theta_{k}\right)+r_{k}^{2}} d V^{*}(t) \\
= & \frac{\alpha}{2} \log \left(1+\frac{4 r_{k} \sin ^{2} \theta_{k} / 2}{\left(1-r_{k}\right)^{2}}\right) \\
& -\frac{1}{2 \pi} \int_{-\pi}^{\pi} \log \left(1+\frac{4 r_{k} \sin \left(t-\theta_{k} / 2\right) \sin \theta_{k} / 2}{\left(1-r_{k}\right)^{2}+4 r_{k} \sin ^{2}\left(t-\theta_{k}\right) / 2}\right) d V^{*}(t) .
\end{aligned}
$$


292

CHRISTIAN POMMERENKE

The part of the last integral that is extended over $\left[-2 \theta_{k}, 2 \theta_{k}\right]$ is

$$
\begin{aligned}
\int_{-2 \theta_{k}}^{2 \theta_{k}} \log (\ldots) d V^{*}(t) & \leqq \int_{-2 \theta_{k}}^{2 \theta_{k}} \log \left(1+\frac{\left|2 t-\theta_{k}\right| \theta_{k}}{\left(1-r_{k}\right)^{2}}\right) d V^{*}(t) \\
& \leqq\left(V^{*}\left(2 \theta_{k}\right)-V^{*}\left(-2 \theta_{l_{i}}\right)\right) \log \left(1+5\left(\frac{\theta_{k}}{1-r_{k}}\right)^{2}\right) .
\end{aligned}
$$

Since $V^{*}(t)$ is continuous at $t=0$ it follows that

$$
\int_{-20_{k}}^{2 o_{k}} \log (\ldots) d V^{*}(t) \leqq o(1) \cdot \log \frac{\theta_{k}}{1-r_{k}}
$$

as $k \rightarrow \infty$. Also, since $\sin \tau \geqq 2 \tau / \pi$ for $0 \leqq \tau \leqq \pi / 2$,

$$
\begin{aligned}
\int_{2 O_{k}}^{\pi} \log (\ldots) d V^{*}(t) & \leqq \int_{2 O_{k}}^{\pi} \log \left(1+\frac{\pi^{2}}{4}\left(2 t-\theta_{k}\right) \theta_{k}\right) d V^{*}(t) \\
& \leqq\left(V^{*}(\pi)-V^{*}(0)\right) \log \left(1+3 \pi^{2} / 4\right)=O(1) .
\end{aligned}
$$

Similarly for the integral over $\left[-\pi,-2 \theta_{l}\right]$. From (9), (10), and (11) we obtain

$$
\begin{aligned}
& \log \left|\frac{f\left(r_{k}\right)}{f\left(r_{k} e^{i\left(r_{k}\right)}\right.}\right| \geqq \frac{\alpha}{2} \log \left(1+{ }_{r_{k} \sin ^{2} \theta_{k} / 2}^{\theta_{k i}{ }^{2}} \cdot\left(\frac{\theta_{k}}{1-r_{k}}\right)^{2}\right) \\
& -o(1) \log \frac{\theta_{l i}}{1-r_{k i}}-O(1) \\
& =(\alpha-o(1)) \log \frac{\theta_{k}}{1-r_{k}}-O(1) .
\end{aligned}
$$

Hence $\theta_{k} /\left(1-r_{k}\right) \rightarrow+\infty$ implies that

$$
\log \left|\frac{f\left(r_{k}\right)}{f\left(r_{k} e^{i 0_{k}}\right)}\right| \rightarrow+\infty
$$

in contradiction to $\left|f\left(r_{k}\right)\right| \leqq M\left(r_{k}\right)=\left|f\left(r e^{i 0_{k}}\right)\right|$. Therefore we have proved that $\theta_{k} /\left(1-r_{k}\right)$ remains bounded if $\alpha>0$.

3. Now let again $\alpha \geqq 0$. By formula (5) in (7),

$$
z \frac{f^{\prime}(z)}{f(z)}=\frac{1}{2 \pi} \int_{-\pi}^{\pi} \frac{1+e^{-i t} z}{1-e^{-i t} z} d V(t)
$$

Therefore (5) implies that

$$
r_{l k} \frac{M^{\prime}\left(r_{k}\right)}{M\left(r_{k}\right)}=\frac{1}{2 \pi} \int_{-\pi}^{\pi} \frac{1+r_{k} e^{i\left(\theta_{k}-t\right)}}{1-r_{k} e^{i\left(l_{k}-t\right)}} d V(t)
$$

Again writing $V(t)=\alpha S(t)+V^{*}(t)$ we obtain

$$
\begin{aligned}
\left(1-r_{k}\right) r_{k} \frac{M^{\prime}\left(r_{k}\right)}{M\left(r_{k}\right)}=\frac{\alpha}{2} & \frac{\left(1+r_{k}\right)\left(1-r_{k}\right)^{2}}{\left(1-r_{k}\right)^{2}+4 r_{k} \sin ^{2} \theta_{k} / 2}+i \alpha \frac{\left(1-r_{k}\right) r_{k} \sin \theta_{k}}{\left(1-r_{k}\right)^{2}+4 r_{k} \sin ^{2} \theta_{k} / 2} \\
& +\frac{1}{2 \pi} \int_{-\pi}^{\pi}\left(1-r_{k}\right) \frac{1+r_{k} e^{i\left(\theta_{k}-t\right)}}{1-r_{k} e^{i\left(\theta_{k}-l\right)}} d V^{*}(t) .
\end{aligned}
$$


The integrand in the last integral tends to 0 as $k \rightarrow \infty$, for each $t \neq 0$. Since $V^{*}(t)$ is continuous for $t=0$, the point $t=0$ has $V^{*}$-measure 0 . Hence the integrand tends to 0 almost everywhere with respect to the monotone function $V^{*}(t)$. Because the integrand is absolutely $\leqq 2$, Lebesgue's theorem on bounded convergence (for Stieltjes integrals) implies that the integral in (13) tends to 0 . If we write

then (13) becomes

$$
\gamma_{k}=\left(\sin \theta_{k} / 2\right) /\left(1-r_{k}\right)
$$

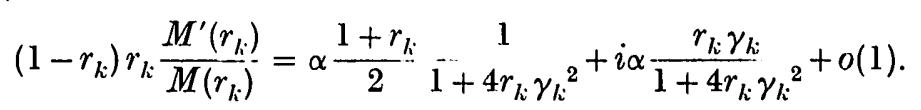

If $\alpha=0$ then it follows at once that $\left(1-r_{k}\right) M^{\prime}\left(r_{k}\right) / M\left(r_{k}\right) \rightarrow 0$. Therefore we may assume that $\alpha>0$. Then we have seen that $\theta_{k} /\left(1-r_{k}\right)$ and thus also $\gamma_{k}$ remain bounded. Since the left side of (14) is real, the second purely imaginary term on the right side tends to 0 . Hence the boundedness of $\gamma_{k}$. implies that $\gamma_{k} \rightarrow 0$. It follows that the first term on the right side of (14) tends to $\alpha$. Hence $\left(1-r_{k i}\right) M^{\prime}\left(r_{k}\right) / M\left(r_{l_{i}}\right) \rightarrow \alpha$, in contradiction to (6).

\section{Estimates of length}

If $f(z)=a_{1} z+\ldots$ is univalent in $|z|<1$ let $L(r)$ denote the length

$$
L(r)=r \int_{0}^{2 \pi}\left|f^{\prime}\left(r e^{i \theta}\right)\right| d \theta
$$

of the image curve $C(r)$ of $|z|=r$. Since the point 0 is enclosed by $C(r)$ it follows that the diameter of $C(r)$ is $\geqq M(r)=\max _{|z|=r}|f(z)|$. Hence $L(r) \geqq 2 M(r)$. It would seem that in general $L(r)$ is very much greater than $M(r)$, due to oscillations of $C(r)$. But it will be shown that this is not quite true if the growth of $M(r)$ is sufficiently large.

Theовем 2. Let $f(z)$ be starlike in $|z|<1$. If the order $\alpha$ of $f(z)$ is positive then there is a $K=K(f)<\infty$ such that

$$
2 M(r) \leqq L(r) \leqq K+\frac{19}{\alpha} M(r) .
$$

Remarks. 1. Since $\pi \alpha$ is the greatest jump of $V(\theta)$, the assumption that $\alpha>0$ means that $V(\theta)$ is not continuous. It can be shown that ' $\alpha>0$ ' is equivalent to the assumption that the image domain of $|z|<1$ contains a sector $\left\{w: \lambda_{1}<\arg w<\lambda_{2}\right\}$ of positive angle $\lambda_{2}-\lambda_{1}$.

2. Since (see for instance (10) vol. I, 286)

$$
P(r)=\sum_{n=1}^{\infty}\left|a_{n}\right| r^{n} \leqq \frac{r}{2} \int_{0}^{2 \pi}\left|f^{\prime}\left(r e^{i n}\right)\right| d \theta=\frac{1}{2} L(r)
$$

and $M(r) \leqq P(r)$, the theorem implies that also $M(r)$ and $P(r)$ have the same growth if $\alpha>0$. 
3. In (8) it was proved that if $f(z)$ is starlike and $M(r) \leqq 1 /(1-r)^{\alpha}(\alpha>0)$ then $L(r) \leqq A(\alpha) /(1-r)^{\alpha}$ where $A(\alpha)$ only depends on $\alpha$. This result is contained in Theorem 2 except for the constant $K$ which may depend on $f$.

We shall need the following known lemma.

ILEMMA. If $F(z)$ is analytic and $\operatorname{Re} F(z)>0$ in $|z|<1$, and if $|F(0)| \leqq 1$, then

and

$$
\frac{1}{2 \pi} \int_{0}^{2 \pi}\left|F\left(r e^{i \theta}\right)\right|^{2} d \theta \leqq \frac{1+3 r^{2}}{1-r^{2}}
$$

$$
\frac{1}{2 \pi} \int_{0}^{2 \pi}\left|F^{\prime}\left(r e^{i \theta}\right)\right| d \theta \leqq \frac{2}{1-r^{2}} .
$$

Proof. Since $\operatorname{Re} F(z)>0$ there is a monotone increasing function $U(t)$ such that

and

$$
\frac{1}{2 \pi} \int_{0}^{2 \pi} d U(t)=\operatorname{Re} F(0)
$$

$$
F(z)=i \operatorname{Im} F(0)+\frac{1}{2 \pi} \int_{0}^{2 \pi} \frac{1+e^{-i t} z}{1-e^{-i t} z} d U(t) .
$$

If $F(z)=c_{0}+c_{1} z+\ldots$ the well-known inequality

$$
\left|c_{n}\right| \leqq 2 \operatorname{Re} F(0) \quad(n \geqq 1)
$$

follows at once. Since $|F(0)| \leqq 1$ we obtain $\left|c_{0}\right| \leqq 1$ and $\left|c_{n}\right| \leqq 2$ for $n \geqq 2$. Therefore

$$
\frac{1}{2 \pi} \int_{0}^{2 \pi}\left|F\left(r e^{i \theta}\right)\right|^{2} d \theta=\sum_{n=0}^{\infty}\left|c_{n}\right|^{2} r^{2 n} \leqq 1+4 \sum_{n=1}^{\infty} r^{2 n}=\frac{1+3 r^{2}}{1-r^{2}} .
$$

Also, (16) implies that

Hence we see that

$$
F^{\prime}(z)=\frac{1}{\pi} \int_{0}^{2 \pi} \frac{e^{-i t}}{\left(1-e^{-i t} z\right)^{2}} d U(t)
$$

$$
\begin{aligned}
\frac{1}{2 \pi} \int_{0}^{2 \pi}\left|F^{\prime}\left(r e^{i \theta}\right)\right| d \theta & \leqq \frac{1}{\pi} \int_{0}^{2 \pi}\left(\frac{1}{2 \pi} \int_{0}^{2 \pi}\left|1-r e^{i(\theta-\bar{l})}\right|^{2} d \theta\right) d U(t) \\
& =\frac{1}{\pi} \int_{0}^{2 \pi} \frac{1}{1-r^{2}} d U(t)=\frac{2}{1-r^{2}} \operatorname{Re} F(0) \leqq \frac{2}{1-r^{2}} .
\end{aligned}
$$

Proof of Theorem 2. Let $F(z)=z f^{\prime}(z) / f(z)$. Then $F(0)=1$. Since $f(z)$ is starlike we have $\operatorname{Re} F(z)>0$. If $h(z)=z f^{\prime}(z)$ then $h(z)=f(z) F(z)$; hence

$$
\begin{aligned}
h^{\prime}(z) & =f^{\prime}(z) F(z)+f(z) F^{\prime}(z) \\
& =\frac{f(z)}{z} F(z)^{2}+f(z) F^{\prime}(z) .
\end{aligned}
$$


Therefore

$$
\frac{1}{2 \pi} \cdot \int_{0}^{2 \pi}\left|h^{\prime}\left(r e^{i \theta}\right)\right| d \theta \leqq r^{-1} M(r) \frac{1}{2 \pi} \int_{0}^{2 \pi}\left|F\left(r e^{i \theta}\right)\right|^{2} d \theta+M(r) \frac{1}{2 \pi} \int_{0}^{2 \pi}\left|F^{\prime}\left(r e^{i \theta}\right)\right| d \theta .
$$

Thus it follows from the Lemma that

$$
\frac{1}{2 \pi} \int_{0}^{2 \pi}\left|h^{\prime}\left(r e^{i \theta}\right)\right| d \theta \leqq M(r) \cdot \frac{1+2 r+3 r^{2}}{r\left(1-r^{2}\right)} .
$$

We can write

$$
L(r)=\int_{0}^{2 \pi}\left|h\left(r e^{i \theta}\right)\right| d \theta=\int_{0}^{2 \pi}\left|\int_{0}^{r} h^{\prime}\left(\rho e^{i \theta}\right) d \rho\right| d \theta .
$$

Hence it follows from (17) that

$$
\begin{aligned}
L(r) & \leqq \int_{0}^{r} \int_{0}^{2 \pi}\left|h^{\prime}\left(\rho e^{i \theta}\right)\right| d \rho d \theta \\
& \leqq 2 \pi \int_{0}^{r} M(\rho) \frac{1+2 \rho+3 \rho^{2}}{\rho\left(1-\rho^{2}\right)} d \rho \leqq 2 \pi \int_{0}^{r} \frac{3 M(\rho)}{\rho(1-\rho)} d \rho .
\end{aligned}
$$

Now we use the hypothesis that $\alpha>0$. By Theorem 1 there is an $r_{0}(f)$ with $\frac{500}{501}<r_{0}<1$ such that

$$
(1-\rho) \frac{M^{\prime}(\rho)}{M(\rho)}>\frac{500}{501} \alpha
$$

for $r_{0} \leqq \rho<1$. Hence (18) shows that, for $r>r_{0}$,

$$
L(r) \leqq 2 \pi \int_{0}^{r_{0}} \frac{3 M(\rho)}{\rho(1-\rho)} d \rho+6 \pi \cdot \frac{501}{500} \cdot \frac{501}{500 \alpha} \int_{r_{0}}^{r} M^{\prime}(\rho) d \rho \leqq K+\frac{19}{\alpha} M(r),
$$

and for $0 \leqq r \leqq r_{0}$ we have $L(r) \leqq K$.

We shall now study the question whether Theorem 2 can be improved. The function

$$
f_{m}(z)=\frac{z}{\left(1-\frac{z}{z^{m}}\right)^{2} / \bar{m}} \quad(m=1,2, \ldots)
$$

maps $|z|<1$ onto the plane cut along the $m$ half-lines

$$
e^{\pi i(2 k-1) / m}\left[2^{-2 / m},+\infty\right] \quad(k=1, \ldots, m) .
$$

Hence $f_{m}(z)$ is starlike, and

$$
L(r) \geqq 2 m(M(r)-1) .
$$

The order of $f(z)$ is $\alpha=2 / m$. Hence

$$
\lim _{r \rightarrow \infty} \inf L(r) / M(r) \geqq 2 m=4 / \alpha .
$$

This inequality shows that the factor $19 / \alpha$ of $M(r)$ on the right side of (15) cannot be replaced by anything better than $4 / \alpha$, at least if $\alpha=2 / m$ $(m=1,2, \ldots)$. 
The hypothesis $\alpha>0$ of Theorem 2 means

$$
\lim _{r \rightarrow 1} \log M(r) / \log \frac{1}{1-r}>0 .
$$

It will now be proved that no weaker assumption implies $L(r)=O(M(r))$.

TheOREM 3. Let $\eta(r)$ be any positive function such that $\eta(r) \rightarrow 0$ as $r \rightarrow 1$. Then there is a starlike function $f(z)$ and a sequence $r_{n} \rightarrow 1$ such that

$$
\log M\left(r_{n}\right) / \log \frac{1}{1-r_{n}} \geqq \eta\left(r_{n}\right)
$$

and

$$
L\left(r_{n}\right) / M\left(r_{n}\right) \rightarrow \infty .
$$

Proof. 1. We shall construct sequences $\lambda_{n}$ and $r_{n}$ with $0<n \lambda_{n}<2^{-n}$ and $r_{n} \rightarrow 1$ such that the functions $f_{0}(z)=z$ and

satisfy

$$
f_{n}(z)=z \prod_{\nu=1}^{n}\left(1-r_{\nu}{ }^{\nu} z^{\nu}\right)^{-\lambda_{\nu}} \quad(n \geqq 1)
$$

$$
\begin{gathered}
\log M_{n}\left(r_{k}\right) \geqq\left(1-2^{-n}\right) \log M_{n-1}\left(r_{k}\right), \\
L_{n}\left(r_{k}\right) / M_{n}\left(r_{k}\right) \geqq\left(1-2^{-n}\right) L_{n-1}\left(r_{k}\right) / M_{n-1}\left(r_{k}\right)
\end{gathered}
$$

$(1 \leqq k \leqq n-1)$, and

$$
\begin{aligned}
\log M_{n}\left(r_{n}\right) & \geqq 2 \eta\left(r_{n}\right) \log 1 /\left(1-r_{n}\right), \\
L_{n}\left(r_{n}\right) & \geqq \frac{1}{2} n M_{n}\left(r_{n}\right) .
\end{aligned}
$$

Here $M_{n}(r)$ is the maximum modulus of $f_{n}(z)$ on $|z|=r$ and $L_{n}(r)$ is the length of the image curve.

2. Suppose the numbers $\lambda_{\nu}$ and $r_{\nu}$ have already been found up to $\nu=n-1$. Let

$$
f_{n}(z)=f_{n-1}(z) \cdot\left(1-r_{n}{ }^{n} z^{n}\right)^{-\lambda_{n}} .
$$

We choose a positive number $\lambda_{n}<2^{-n} / n$ so small that (19) and (20) hold uniformly in $r_{n}$ for $0<r_{n}<1$. It follows from geometric considerations that the function $z\left(1-r_{n}{ }^{n} z^{n}\right)^{-\lambda_{n}}$ maps $|z|=r_{n}$ onto a curve of length $>2 n\left[\left(1-r_{n}{ }^{2 n}\right)^{-\lambda_{n}}-1\right]$. We can choose $r_{n}\left(>r_{n-1}\right)$ so large that the following four conditions are satisfied:

$$
\begin{gathered}
L_{n}\left(r_{n}\right)>n r_{n}\left(1-r_{n}{ }^{2 n}\right)^{-\lambda_{n},} \\
r_{n}\left(1-r_{n}{ }^{2 n}\right)^{-\lambda_{n}}>\frac{1}{2} M_{n}\left(r_{n}\right), \\
M_{n}\left(r_{n}\right)>\frac{1}{2}\left(1-r_{n}{ }^{2 n}\right)^{-\lambda_{n},} \\
\frac{1}{2}\left(1-r_{n}{ }^{2 n}\right)^{-\lambda_{n}}>\left(1-r_{n}{ }^{n}\right)^{-2 \eta\left(r_{n}\right)} .
\end{gathered}
$$

Then (21) follows from (25) and (26), and (22) from (23) and (24). 
3. Let

$$
f(z)=\lim _{n \rightarrow \infty} f_{n}(z)=z \prod_{\nu=1}^{\infty}\left(1-r_{\nu}{ }^{\nu} z^{\nu}\right)^{-\lambda_{\nu}}
$$

Since $\Sigma \nu \lambda_{\nu}<\Sigma 2^{-\nu}<2$, computation shows that $f(z)$ is starlike. For fixed $k \geqq 1$ and $n>k$ it follows from (19) that

$$
\log M_{n}\left(r_{k}\right) \geqq\left(1-2^{-n}\right) \ldots\left(1-2^{-k-1}\right) \log M_{k}\left(r_{k}\right)>\frac{1}{2} \log M_{k}\left(r_{k}\right) ;
$$

hence, by (21),

$$
\log M\left(r_{k}\right)=\lim _{n \rightarrow \infty} M_{n}\left(r_{k}\right) \geqq \eta\left(r_{k}\right) \log 1 /\left(1-r_{k}\right) .
$$

It follows from (20) that

$$
L_{n}\left(r_{k}\right) / M_{n}\left(r_{k}\right)>\sum_{2}^{1} L_{k}\left(r_{k}\right) / M_{k}\left(r_{k_{i}}\right)
$$

and therefore, by (22),

$$
L\left(r_{k}\right) / M\left(r_{k}\right)>k / 4
$$

\section{Coefficient problems}

Let $f(z)=\sum_{n=1}^{\infty} a_{n} z^{n}$ be univalent in $|z|<1$. Recently, Hayman (6) has shown that

$$
|| a_{n+1}|-| a_{n}||<A\left|a_{1}\right|
$$

for some absolute constant $A$. In the special case that $f(z)$ is starlike this had been proved by Golusin (2) (with $A<100$ ). In the general case, Biernacki (1) had earlier obtained $A\left|a_{1}\right|(\log (n+1))^{\frac{1}{2}}$ as an upper bound.

A function $f(z)$ analytic in $|z|<1$ is called close-to-convex if there is a starlike function $g(z)=b_{1} z+\ldots$ such that

$$
\operatorname{Re} \frac{z f^{\prime}(z)}{g(z)}>0
$$

for $|z|<1$. This class of functions was introduced by Kaplan (7). He showed that every close-to-convex function is univalent.

The starlike functions form a subclass of the class of close-to-convex functions (with $g(z) \equiv f(z)$ ). Another subclass is formed by the functions convex in one direction, introduced by Robertson (9). These are the functions for which the intersection of the image region with each line of a certain fixed direction is either empty or one interval. A function is convex in one direction if and only if the starlike function in (27) has the form

$$
g(z) \equiv \begin{gathered}
b_{1} z \\
\left(1-e^{-i \theta_{1}} z\right)\left(1-e^{-i \theta_{2}} z\right)
\end{gathered}
$$

with real $\theta_{1}$ and $\theta_{2}$ and complex $b_{1}$.

Theorem 4. Let $f(z)=\sum_{n=1}^{\infty} a_{n} z^{n}$ be close-to-convex in $|z|<1$. Then

$$
|| a_{n+1}|-| a_{n}||<{ }_{4}^{3} e^{2}\left|a_{1}\right| \text {. }
$$


If the starlike function $g(z)$ in (27) does not have the form (28) then there is $a \delta=\delta(g)>0$ such that

$$
\left|a_{n+1}\right|-\left|a_{n}\right|=O\left(\frac{1}{n^{\delta}}\right) .
$$

Remarks. 1. For the special case that $f(z)$ is convex in one direction Professor M. S. Robertson communicated to me a proof that

$$
-5 \leqq\left|a_{n+1}\right|-\left|a_{n}\right|<4 \text {, }
$$

which is a little better than the above estimate $\left(\frac{3}{4} e^{2} \approx 5 \cdot 54\right)$.

2. The functions excluded in the second part of Theorem 4 are those functions close-to-convex in one direction for which only a $g$ of the form (28) can be chosen. 'i'o see that there are functions convex in one direction for which not even $\left|a_{n+1}\right|-\left|a_{n}\right| \rightarrow 0$ holds we can take $f(z) \equiv g(z)$, of the form (28). Another example is given by the odd function which maps $|z|<1$ onto the complement of $\{|\operatorname{Re} v| \leqq 1,|\operatorname{Im} w| \geqq 1\}$.

CoRollary 1. Let $f(z)$ be starlike in $|z|<1$. Then either

$$
f(z) \equiv \frac{a_{1} z}{\left(1-e^{-i \theta_{1}} z\right)\left(1-e^{-i \theta_{2}} z\right)},
$$

or there is a $\delta=\delta(f)>0$ such that

$$
\left|a_{n+1}\right|-\left|a_{n}\right|=O\left(\frac{1}{n^{\delta}}\right)
$$

The second part of Theorem 4 can be generalized.

Theorem 5 . Let $m$ be an integer $\geqq 1$, and let $f(z)$ be close-to-convex in $|z|<1$. Let $\operatorname{Re}\left[z f^{\prime}(z) ! g(z)\right]>0$ for a starlike function

$$
g(z) \neq b_{1} z \prod_{k=1}^{m+1}\left(1-e^{-i o_{k}} z\right)^{-2 /(m+1)} \text {. }
$$

Then there are complex numbers $c_{k}(k=0, \ldots, m)$ with $\left|c_{0}\right|=\left|c_{m}\right|=1$, and $a \delta>0$ depending only on $m$ and $g$, such that

$$
c_{0} n^{2} a_{n}+c_{1}(n+1)^{2} a_{n+1}+\ldots+c_{m}(n+m)^{2} a_{n+m}=O\left(n^{2 /(m+1)+1-\delta}\right) .
$$

Proof of Theorem 4. We may assume that $\left|a_{1}\right|=\left|f^{\prime}(0)\right|=1$ and $\left|g^{\prime}(0)\right|=1$. Because of $(27)$ and because $g(z)$ is starlike, the functions

$$
F(z)=\frac{z f^{\prime}(z)}{g(z)}, \quad G(z)=\frac{z g^{\prime}(z)}{g(z)}
$$

have positive real part, and $\left|F^{\prime}(0)\right|=G^{\prime}(0)=1$. Since $g(z)$ is univalent we can chorse a $\zeta=\zeta(r)$ with $|\zeta|=r$ such that

$$
Q(r)=\max _{|z|=r}|(z-\zeta) g(z)| \leqq \frac{2 r^{2}}{1-r^{2}}
$$


((3) 162). If $h(z)=z f^{\prime}(z)$ then, by $(30)$,

$$
h^{\prime}(z)=\frac{d}{d z}(g(z) F(z))=z^{-1} g(z)\left(G(z) F(z)+z F^{\prime}(z)\right) .
$$

With $z=r e^{i 0}$ we therefore obtain

$$
\frac{1}{2 \pi} \int_{0}^{2 \pi}\left|(z-\zeta) h^{\prime}(z)\right| d \theta \leqq r^{-1} Q(r)\left(\frac{1}{2 \pi} \int_{0}^{2 \pi}|G(z) F(z)| d \theta+\frac{r}{2 \pi} \int_{0}^{2 \pi}\left|F^{\prime}(z)\right| d \theta\right) .
$$

By the Schwarz inequality,

$$
\frac{1}{2 \pi} \int_{0}^{2 \pi}|G(z) F(z)| d \theta \leqq\left(\frac{1}{2 \pi} \int_{0}^{2 \pi}|G(z)|^{2} d \theta \cdot \frac{1}{2 \pi} \int_{0}^{2 \pi}|F(z)|^{2} d \theta\right)^{\frac{1}{2}} .
$$

Hence application of the lemma to $G(z)$ and $F(z)$ shows, with (31), that

$$
\begin{aligned}
\frac{1}{2 \pi} \int_{0}^{2 \pi}\left|(z-\zeta) h^{\prime}(z)\right| d \theta & \leqq r^{-1} Q(r)\left(\frac{1+3 r^{2}}{1-r^{2}}+\frac{2 r}{1-r^{2}}\right) \\
& \leqq \frac{2 r\left(1+2 r+3 r^{2}\right)}{\left(1-r^{2}\right)^{2}}
\end{aligned}
$$

Since $h^{\prime}(z)=\frac{d}{d z}\left(z f^{\prime}(z)\right)=\sum_{1}^{\infty} n^{2} a_{n} z^{n-1}$ we see that

$$
\begin{aligned}
(z-\zeta) h^{\prime}(z) & =\sum_{1}^{\infty} n^{2} a_{n} z^{n}-\sum_{1}^{\infty} n^{2} a_{n} \zeta z^{n-1} \\
& =-a_{1} \zeta+\sum_{1}^{\infty}\left(n^{2} a_{n}-(n+1)^{2} a_{n+1} \zeta\right) z^{n}
\end{aligned}
$$

Therefore (32) implies that

$$
\left|n^{2} a_{n}-(n+1)^{2} a_{n+1} \zeta\right| \leqq \frac{1}{r^{n}} \frac{2 r\left(1+2 r+3 r^{2}\right)}{\left(1-r^{2}\right)^{2}} .
$$

We take $r=r_{n}=(n /(n+1))^{2}$. Because $|\zeta|=r_{n}$ it follows that

$$
\begin{aligned}
n^{2} \| a_{n}|-| a_{n+1} \mid & \leqq \frac{1}{r_{n}{ }^{n}} \frac{1+2 r_{n}+3 r_{n}^{2}}{\left(1+r_{n}\right)^{2}} \cdot \frac{2 r_{n}}{\left(1-r_{n}\right)^{2}} \\
& <\frac{3}{4}\left[\left(1+\frac{1}{n}\right)^{n} \frac{2 n+2}{2 n+1}\right]^{2} n^{2}<\frac{3}{4} e^{2} n^{2} .
\end{aligned}
$$

This proves the first part of Theorem 4.

The second part follows from Theorem 5 . We have

$$
(n+1)^{2}|| a_{n}|-| a_{n+1} \| \leqq\left|n^{2}\right| a_{n}\left|-(n+1)^{2}\right| a_{n+1}||+(2 n+1)\left|a_{n}\right| .
$$

By Theorem 5 the first term is $O\left(n^{2-\delta^{\prime}}\right)$ for some $\delta^{\prime}>0$. From the assumption about $g$ it follows that the starlike function $g$ has an order $\alpha_{1}<2$. Hence $g(z)=O\left((1-r)^{-\beta}\right)$, for some $\beta$ with $1<\beta<2$. Since $\operatorname{Re} F(z)>0$, so that $F(z)=O\left((1-r)^{-1}\right)$, and $z f^{\prime}(z)=g(z) F(z)$, we see that $f^{\prime}(z)=O\left((1-r)^{-\beta-1}\right)$. Therefore $f(z)=O\left((1-r)^{-\beta}\right)$. Since $f(z)$ is univalent and $\beta>1$ it follows 
that $a_{n}=O\left(n^{\beta-1}\right)((5) 46)$. Therefore (33) implies that

$$
(n+1)^{2}|| a_{n}|-| a_{n+1} \|=O\left(n^{2-\delta^{\prime}}\right)+O\left(n^{\beta}\right)=O\left(n^{2-\delta}\right)
$$

for $\delta=\min \left(\delta^{\prime}, 2-\beta\right)>0$.

Proof of Theorem 5. 1. Let

$$
V(\theta)=\lim _{r \rightarrow 1} \arg g\left(r e^{i \theta}\right) .
$$

Let $\pi \alpha_{k}(k=1, \ldots, m+1)$ be the $m+1$ greatest jumps of the monotone function $V(\theta)$, and let

$$
\alpha_{1} \geqq \alpha_{2} \geqq \ldots \geqq \alpha_{m+1}
$$

Since $V(\theta)$ is monotone,

$$
\alpha_{1}+\ldots+\alpha_{m}+\alpha_{m+1} \leqq \frac{1}{2 \pi} \int_{0}^{2 \pi} d V(\theta)=2,
$$

with equality only if $V(\theta)$ is constant except for the jumps $\pi \alpha_{k i}$. Let $\theta_{k}$ be the point in $0 \leqq \theta<2 \pi$ at which $V(\theta)$ has the jump $\pi \alpha_{l i}$.

2. We have now to distinguish two cases.

(i) Suppose that $\alpha_{1}<1+2 /(m+1)$. It follows from (34) and (35) that

$$
\alpha_{k}<1+2 /(m+1) \text { and }(m+1) \alpha_{m+1} \leqq \alpha_{1}+\ldots+\alpha_{m+1} \leqq 2 ;
$$

hence $\alpha_{m+1} \leqq 2 /(m+1)$. If we had equality then $V(\theta)$ would be constant except for jumps of height $2 \pi /(m+1)$ at $\theta=\theta_{k}(k=1, \ldots, m+1)$. But then $g(z)$ would have the form excluded in Theorem 5 . Therefore $\alpha_{m+1}<2 /(m+1)$. We define

$$
g^{*}(z)=g(z) \psi(z) \text { with } \quad \psi(z)=\prod_{k=1}^{m}\left(1-e^{-i 0_{k}} z\right) .
$$

Let $S(\theta)$ be the function which is constant in $-\infty<\theta<\infty$ except for positive jumps of height $\pi$ at the multiples of $2 \pi$. Let

$$
V^{*}(\theta)=V(\theta)-\sum_{k=1}^{m} S\left(\theta-\theta_{k_{i}}\right)
$$

'Then all positive jumps of $V^{*}(\theta)$ have height

$$
\leqq \pi \max \left(\alpha_{1}-1, \ldots, \alpha_{m}-1, \alpha_{m+1}\right)<2 \pi /(m+1) .
$$

(ii) Suppose that $\alpha_{1} \geqq 1+2 /(m+1)$. It follows that $m \geqq 2$ because otherwise $\alpha_{1}=2$, and $g(z)$ would be of the excluded form. By (35) we obtain $\alpha_{k i} \leqq 2-\alpha_{1}<1$ for $k \geqq 2$. Also by (35),

$$
(m-1) \alpha_{m} \leqq \alpha_{2}+\ldots+\alpha_{m} \leqq 2-\alpha_{1} \leqq 1-\frac{2}{m+1}=\frac{m-1}{m+1}
$$

hence $\alpha_{m} \leqq 1 /(m+1)<2 /(m+1)$. We define

$$
g^{*}(z)=g(z) \psi(z) \quad \text { with } \quad \psi(z)=\left(1-e^{-i 0_{1}} z\right)^{2} \prod_{k=2}^{m-1}\left(1-e^{-i \theta_{k}} z\right) .
$$


Then all positive jumps of

$$
V^{*}(\theta)=V(\theta)-2 S\left(\theta-\theta_{1}\right)-\sum_{k=2}^{m-1} S\left(\theta-\theta_{l i}\right)
$$

have height $\leqq \pi \max \left(\alpha_{1}-2, \alpha_{2}-1, \ldots, \alpha_{m-1}-1, \alpha_{m}\right)=\pi \alpha_{m}<2 \pi !(m+1)$.

3. The functions $V^{*}(\theta)$ defined by (37) or (39) have variation $\leqq(m+2) \pi$ over every interval of length $2 \pi$. We have proved that in either case the positive jumps of $V^{*}(\theta)$ have height $<2 \pi /(m+1)$. Also, it follows from (37) and (39) that $V^{*}(\theta)-(1-m / 2) \theta$ has the period $2 \pi$. Using (7) we obtain

$$
g^{*}(z)=b_{1} z \exp \left(\frac{1}{\pi} \int_{0}^{2 \pi} \log \frac{1}{1-e^{-\pi} z} d V^{*}(t)\right) .
$$

This equation implies (see the proof of Theorem $l$ in (7)) that

$\log \left|g^{*}\left(r e^{i 0}\right)\right|-\log r\left|b_{1}\right|$

$$
\begin{aligned}
& =\frac{1}{2 \pi} \int_{-\pi}^{\pi} \begin{array}{l}
2 r \sin \tau \\
l-2 r \cos \tau+r^{2}
\end{array}\left(V^{*}(\theta+\tau)-\left(1-\frac{m}{2}\right) \tau\right) d \tau \\
& =\frac{1}{2 \pi} \int_{0}^{\pi} \ddot{1-2} \frac{2 r \sin \tau}{r \cos \tau+r^{2}}\left(V^{*}(\theta+\tau)-V^{*}(\theta-\tau)-2\left(1-\frac{m}{2}\right) \tau\right) d \tau .
\end{aligned}
$$

For given $0<\eta<\pi / 2$ we split the integral into an integral over $[0, \eta]$ and one over $[\eta, \pi]$. The second integral is

$$
\leqq \frac{3(m+2) \pi r}{1-2 r \cos \eta+r^{2}} \text {. }
$$

The integral over $[0, \eta]$ is either negative or

$$
\leqq \frac{1}{\pi} \log \frac{1+r}{1-r} \cdot \sup _{0 \leqq \tau \leqq \eta}\left(V^{*}(\theta+\tau)-V^{*}(\theta-\tau)-(2-m) \tau\right) .
$$

Since the positive jumps of $V^{*}(t)$ are $<2 \pi /(m+1)$, it follows that there are a $\delta>0$ and an $\eta>0$ such that

$$
\sup _{0 \leqq \tau \leqq \eta}\left(V^{*}(\theta+\tau)-V^{*}(\theta-\tau)-(2-m) \tau\right)<\pi\left(\frac{2}{m+1}-\delta\right)
$$

for all $\theta$. Hence

$$
\log \left|g^{*}\left(r e^{i \eta}\right)\right| \leqq\left(\frac{2}{m+1}-\delta\right) \log \frac{1}{1-r}+K_{1}
$$

where $K_{1}$ does not depend on $r$.

4. As in the proof of Theorem 4, let $h(z)=z f^{\prime}(z)$. Then again

$$
h^{\prime}(z)=z^{-1} g(z)\left(G(z) F(z)+z F^{\prime}(z)\right) .
$$

If $\psi(z)$ is defined by (36) or (38) then (with $z=r e^{i 0}$ )

$$
\begin{aligned}
\frac{1}{2 \pi} \int_{0}^{2 \pi}\left|\psi(z) h^{\prime}(z)\right| d \theta & =\frac{1}{2 \pi r} \int_{0}^{2 \pi}\left|g^{*}(z)\right|\left|G(z) F(z)+z F^{\prime}(z)\right| d \theta \\
& \leqq Q(r) \frac{1+2 r+3 r^{2}}{r\left(1-r^{2}\right)}
\end{aligned}
$$


by the lemma where, from (40),

$$
Q(r)=\max _{|\varepsilon|=r}\left|g^{*}(z)\right|=O\left((1-r)^{-2 /(m+1)+\delta}\right) .
$$

Hence we see that

$$
\frac{1}{2 \pi} \int_{0}^{2 \pi}\left|\psi(z) h^{\prime}(z)\right| d \theta=O\left((1-r)^{-2 /(m+1)-1+\delta}\right) .
$$

By (36) and (38) we can write

$$
\psi(z)=\sum_{i=0}^{m} c_{k} z^{m-k}
$$

with $\left|c_{0}\right|=\left|c_{m}\right|=1$. In the power series expansion of

$$
\psi(z) h^{\prime}(z)=\sum_{k=1}^{m} c_{k} z^{m-k} \sum_{\nu=1}^{\infty} \nu^{2} a_{\nu} z^{\nu-1}
$$

the coefficient of $z^{n+m-1}$ is

Therefore (41) implies that

$$
\sum_{k=0}^{m} c_{k}(n+k)^{2} a_{n+k}
$$

$$
\left|\sum_{k=0}^{m} c_{k}(n+k)^{2} a_{n+k}\right| \leqq K_{2} r^{-n-m+1}(1-r)^{-2 /(m+1)-1+\delta},
$$

where $K_{2}$ does not depend on $n$ and $r$. If we choose $r=1-1 / n$, Theorem 5 follows.

Theorem 6. Let $f(z)=\sum_{1}^{\infty} a_{n} z^{n}$ be starlike in $|z|<1$. Let $m=1,2, \ldots$ If there is a sequence $\left\{n_{\nu}\right\}$ satisfying

$$
\begin{aligned}
& n_{\nu+1}-n_{\nu}=O\left(n_{\nu}{ }^{\epsilon}\right) \text { for every } \epsilon>0 \text {, if } m=1 \text {, } \\
& n_{\nu+1}-n_{\nu}=o\left(\log n_{\nu}\right) \quad \text { if } m>1 \text {, }
\end{aligned}
$$

such that

$$
\max _{0 \leqq k \leqq m-1}\left|a_{n_{\nu}+k}\right|=O\left(n_{\nu}^{2 /(m+1)-1-\eta^{\prime}}\right)
$$

for some $\eta^{\prime}>0$, then either

$$
f(z)=a_{1} z \prod_{k=0}^{m} \frac{1}{\left(1-e^{-i o_{k}} z\right)^{2 /(m+1)}},
$$

or there is an $\eta>0$ such that for all $n$,

$$
a_{n}=O\left(n^{2 /(m+1)-1-\eta}\right) \text {. }
$$

Remark. The coefficients of $f(z)=z\left(1-z^{m+1}\right)^{-2 /(m+1)}$ satisfy $a_{n}=0$ for $n \neq 1(\bmod (m+1))$ and $a_{n} \sim$ const. $n^{2 /(m+1)-1}$ for $n \equiv 1(\bmod (m+1))$. Therefore the assumption that $f(z)$ shall not have the form (45) cannot be dropped. 
ON STARLIKE AND CLOSE.TO-CONVEX FUNCTIONS 303

Finally we shall prove that if a starlike function has not too few gaps then either the coefficients are very regularly distributed or they are small.

Corollary 2. Let $f(z)$ be starlike in $|z|<$. I. If there is a sequence $\left\{n_{v}\right\}$ satisfying (42) such that $a_{n_{\nu}}=0$ then either

(i) there is a $q>0$ such that $\left\{a_{n}\right\}$ has the period $q$ and $a_{n}=0$ exactly if $n \equiv 0(\bmod q)$, or

(ii) there is an $\eta>0$ such that $a_{n}=O\left(n^{-1}\right)$.

Proof of Theorem 6. 1. Let first $m=1$, and let us assume that (45) is not true. From Corollary 1 we obtain $\left|a_{\mu}\right|-\left|a_{\mu+1}\right|=O\left(\mu^{-\delta}\right)$ for some $\delta>0$. If $n$ is given let $n_{\nu}$ be such that $n_{\nu-1}<n \leqq n_{\nu}$. Then

$$
\begin{aligned}
\left|a_{n}\right| & \leqq\left|a_{n}\right|-\left|a_{n+1} \|+\ldots+\right|\left|a_{n_{\nu}-1}\right|-\left|a_{n_{\nu}}\right|+\left|a_{n_{\nu}}\right| \\
& \leqq\left(n_{\nu}-n_{\nu-1}\right) \max _{\mu \geqq n}\left\|a_{\mu}|-| a_{\mu+1}\right\|+\left|a_{n_{\nu}}\right| .
\end{aligned}
$$

Because of (42) we have $n_{\nu}-n_{\nu-1}=O\left(n_{\nu-1}^{\delta / 2}\right)$. Therefore (44) implies that

$$
a_{n}=O\left(n_{\nu-1}^{\delta / 2}\right) O\left(n^{-\delta}\right)+O\left(n_{\nu}^{-\eta^{\prime}}\right)=O\left(n^{-\eta}\right),
$$

with $\eta=\min \left(\eta^{\prime}, \delta / 2\right)$.

2. Let now $m>1$, and let (45) be not true. By Theorem 5 (with $g(z) \equiv f(z))$ there are a $\delta>0$ and a $K$ such that

$$
n^{2}\left|a_{n}\right| \leqq \sum_{k=1}^{m}\left|c_{k}\right|(n+k)^{2}\left|a_{n+k}\right|+K n^{2 /(m+1)+1-\delta} .
$$

If $C=\max _{1 \leqq k \leqq m}\left|c_{k}\right|$ it follows by induction that

$$
\begin{aligned}
n^{2}\left|a_{n}\right| \leqq C(1 & +C)^{p} \sum_{k=1}^{m}(n+k+p)^{2}\left|\alpha_{n+k+p}\right| \\
& +K(1+C)^{p}(n+p)^{2 /(m+1)+1-\delta}
\end{aligned}
$$

for every $p=0,1, \ldots$. For given $n$ let $n_{\nu-1} \leqq n<n_{\nu}$. We take $p=n_{\nu}-n-1$ in (46). Together with (44) we thus obtain

$$
n^{2}\left|a_{n}\right|=(1+C)^{n_{\nu}-n_{\nu-1}}\left(O\left(n_{\nu}^{2 /(m+1)+1-\eta^{\prime}}\right)+O\left(n^{2 /(m+1)+1-\delta}\right)\right) .
$$

By (43) we have

$$
\log (1+C)^{n_{\nu}-n_{\nu-1}}=o\left(\log n_{\nu}\right) .
$$

Therefore it follows from (47) that

$$
n^{2}\left|a_{n}\right|=O\left(n^{2 /(m+1)+1-\eta}\right),
$$

with $\eta=\min \left(\eta^{\prime} / 2, \delta / 2\right)>0$.

Proof of Corollary 2. Let us assume that (ii) is not true. 'T'hen it follows from Theorem 6 that

$$
f(z)=\frac{a_{1} z}{\left(1-e^{-i \theta_{1}} z\right)\left(1-e^{-i \theta_{2}} z\right)}=\frac{a_{1}}{e^{-i \theta_{1}}-e^{-i \hat{O}_{2}}}\left(\frac{1}{1-e^{-i \hat{O}_{1}} z}-\frac{1}{1-e^{-i \hat{O}_{z}} z}\right) .
$$


(It is not possible that $\theta_{1}=\theta_{2}$ because then $\left|a_{n}\right|=n \neq 0$.) The coefficients of this function are

$$
a_{n}=\text { const. } e^{-i 0_{1} n}\left(1-e^{i\left(O_{1}-O_{2}\right) n}\right) .
$$

If $\left(\theta_{1}-\theta_{2}\right) / 2 \pi$ were irrational then $a_{n} \neq 0$ for all $n$. If $q$ is the lowest positive denominator of $\left(\theta_{1}-\theta_{2}\right) / 2 \pi$ then (i) follows.

\section{REFERENCES}

1. M. Biernacki, 'Sur les coefficientes de Taylor des fonctions univalentes', Bull. Acad. Polon. S'ci., Cl. III, 4 (1956) 5-8.

2. G. M. Golusin, 'Über die Verzerrungssütze und die Koeffizienten schlichter Funktionen', Mat. Sbornik 19 (1946) 183-202 (Russian, Gorman summary).

3. - Geometrische Funktionentheorie (Berlin, 1957).

4. W. K. Hayman, 'A characterization of the maximum modulus of a function regular at the origin', J. Analyse Math. 1 (1951) 135-54.

5. - Multivalent functions (Cambridge, 1958).

6. 'On successive coefficients of univalent functions', J. London Math. Soc. (to apppear).

7. W. Kaplan, 'Close-to-convex schlicht functions', Michigan Math. J. 1 (1952) 169-85.

8. Сh. Pommerenke, 'On starlike and convex functions', J. London Math. Soc. 37 (1962) 209-24.

9. M. S. Robertson, 'Analytic functions starlike in one direction', American $J$. Math. 58 (1936) 465-72.

10. A. Zyamund, Trigonometric series, Vol. I, 2nd edn (Cambridge, 1959).

\section{University of Michigan}

